\title{
Ethics in Medical Practice
}

Medical ethics is a system of moral principles that apply values to the practice of clinical medicine and in scientific research. Medical ethics is based on a set of values that professionals can refer to in the case of any confusion or conflict. These values include the respect of autonomy, non-maleficence, beneficence and justice ${ }^{1}$. Such tenets may allow doctors, care provider, and families to create a treatment plan and work towards the same common goal without any conflict ${ }^{2}$. It is important to note that these four values are non-hierarchical, meaning not one principle routinely trumps the other ${ }^{3}$. It provides standards of behavior and decision-making that enables physicians to know what is expected of them by their colleagues, their patients and society in general. It also focuses on major social issues like abortion, organ transplantation and euthanasia.

The term medical ethics first dates back to 1803, when English author and physician Thomas Percival publish a document describing the requirements and expectations of medical professionals within medical facilities. The code of ethics was then adapted in 1847, relying heavily on Percival's words ${ }^{4}$. Over the years in 1903, 1912 and 1947, revisions have been made to the original document ${ }^{4}$. Since then practice of Medical Ethics is widely accepted and practiced throughout the world ${ }^{3}$. World Medical Association at its third general assembly in Geneva, September 1948 proposed certain codes of ethics in the form of an oath to be taken by all members of the medical profession at the time of entering into the profession. A year later the association adopted this code of ethics which is popularly known as the international code of medical ethics.

Bangladesh Medical and Dental Council is the body to regulate the conduct of Medical Professionals of Bangladesh. After graduation they have to register themselves with the council. They have to agree to abide by the codes of declaration.

During the past decade, the traditional medical ethical principle of confidentiality, that is, the physician's duty to protect the patient's personal health information, has come into increasing conflict with a perceived need for health information databases serving administrative, planning and research purposes. Computerization has greatly facilitated the establishment and linking of such databases and, thereby, has made breaches of confidentiality much easier.

Physicians have strong reasons for preserving confidentiality. In order to receive medical care, patients have to reveal personal information to physicians who is stranger to them- information that they would not want anyone else to know. They must have good reason to trust their physicians not to divulge this information. The basis of this trust is the ethical and legal standards of confidentiality that physicians and other health care professionals are expected to uphold. Without an understanding that their disclosures will be kept secret, patients may with-hold personal information. This can hinder physicians in their efforts to provide effective interventions or to attain important public health goals.

In an increasingly globalized world, the need for international standards of medical ethics has never been greater. Clinical trials often involve researchers and patients in many different countries, and the same ethical requirements must apply in all cases if the trials are to receive approval. Outside the research context, there remain differences in how general ethical rules are applied, for example, with regard to informed consent. In some countries, patients must be told all they need and want to know about their medical condition and the options for treatment so that they can make informed decisions, also for other countries, it is felt that terminally ill patients should not be informed of their prognosis. However, as both physicians and patients migrate in large numbers from country to country, either temporarily or permanently, there is an increasing need to recognize the fundamental similarities of the principles of medical ethics everywhere and to reconcile the different applications of these principles.

The many ethical statements and resolutions of the WMA are proof that it is possible to reach international consensus on the most difficult ethical issues in medical practice. Such consensus does not always come easily; it is often the result of considerable discussion and consultation. Moreover, the policies require periodic review, since both medical science and ethics do evolve, sometimes at a very rapid pace. The WMA recently 
instituted a systematic review process to ensure that its policies remain up-to-date ${ }^{5}$.

At present Bangladesh is going through social transformation, economic growth and increasing disparity among people, widening of gap between poor and rich, increasing literacy, empowerment of women, increased health awareness, increasing life expectancy, decreasing infant and maternal mortality. ${ }^{6}$ There is a change of life style of the people and family dynamics, health seeking behavior, improvement of information technology and rapid urbanization. These are the challenges in the health service.

In Bangladesh, medical services are provided by public and private sectors. In public sector there are scarcities of amenities and beds. It is overcrowded and the physicians have to face the ethical dilemma in their choice of treatment and priority. In private sector it is costly and beyond the reach of poor people. There is lack of good monitoring and proper laws.

In teaching Medical Ethics colonial legacy is being followed. It is taught in the department of Forensic Medicine emphasizing negligence and malpractice; but medical students mostly follow the professionals conduct of their seniors rather than learning through text books and class instructions.
It is time for restructuring the guidelines of medical ethics and incorporates them in the undergraduate medical curriculum, strengthening the Medical Council, sensitizing the physicians and upgrading the relevant laws.

[J Shaheed Suhrawardy Med Coll 2017; 9(2): 41-42] DOI: http://dx.doi.org/10.3329/jssmc.v9i2.37260

Prof. Md. Mustafizur Rahman, Professor of Surgery, Shaheed Suhrawardy Medical College, Dhaka

Prof. ABM Muksudul Alam, Principal, Shaheed Suhrawardy Medical College, Dhaka

\section{References}

1. Beauchamp, J. (2013). "Principles of Biomedical Ethics". Principles of Biomedical Ethics. 7.

2. Weise, Mary (2016). "Medical Ethics Made Easy". Professional Case Management. 21 (2): 88-94. doi:10.1097/ ncm. 0000000000000151. PMID 26844716. Archived from the original on 2017-04-01.

3. "Bioethic Tools: Principles of Bioethics". depts. washington.edu. Archivedfrom the original on 2017-05-10. Retrieved 2017-03-21.

4. Riddick, Frank (2003). "The Code of Medical Ethics of the American Medical Association”. The Ochsner Journal. 5 (2): 6-10. PMC 3399321/ ¿. PMID 22826677.

5. www.wma.net/e/ethicsunit/polices.htm

6. Mahbub RE. 'Medical Ethics in Bangladesh'. Bangladesh Medical Journal. 2012; 41(1): 7. 